\title{
La France en quête de confiance : la construction des émotions dans les vœux des hommes politiques pour la nouvelle année
}

\author{
France in the search of confidence: constructing emotions \\ in politicians' New Year's wishes
}

\author{
Monika Kostro \\ Université de Varsovie \\ mkostro@uw.edu.pl
}

\begin{abstract}
With the development of political communication over the Internet, a growing number of the French politicians are posting online their New Year's Messages, which have been so far a genre of political discourse reserved for the President. These speeches have a similar pattern, which apart from wishes include an evaluation of the passing year and plans for the future. This is so, because the New Year's Messages have primarily a persuasive function, and serve the purpose of strengthening the addressees' group identity by reminding them of common values. However, while the President's speech is strongly ritualized and emotionally balanced - as it is addressed to the whole nation - the online speeches are not subject to certain conventions, which allows the politicians to adjust the tone of their speech to the expectations of their supporters. Therefore a whole range of feelings is applied - from the fear of France falling, to national pride - depending on the political aims which are to be achieved. This article makes an attempt at answering the question in which way these emotions are constructed within the speeches, and which persuasive function they embody. The author adopts the perspective of argument analysis in discourse (Amossy, 2000; Plantin, 2011), which perceives emotions as a rhetoric means (pathos) whose aim is to influence the addressee.
\end{abstract}

Keywords: emotions, pathos, New Year's Messages, discourse analysis, persuasion

Sous la $\mathrm{V}^{\mathrm{e}}$ République, les vœux présidentiels diffusés à la télévision le 31 décembre sont devenus un véritable rituel politique dont la forme, à quelques exceptions près, n'a pas changé depuis Charles de Gaulle qui en a inventé la formule (Leblanc, 2008 ; Patier, 2011 : 9-16). Cependant, avec le développement de la communication politique en ligne, les vœux aux Français ont cessé d'être 
l'apanage du chef de l'État. De plus en plus d'hommes et de femmes politiques publient leurs propres messages de Nouvel An sur leurs blogs, sur Facebook ou sur Twitter. Diffusés d'habitude avant l'allocution du président de la République, ils sont largement commentés par les médias, à l'affût de l'actualité politique en période de " trêve des confiseurs ", et se transforment, à leur tour, en une nouvelle pratique rituelle. Ce qui est nouveau, ce n'est pas seulement la pluralité des vœux de Nouvel An en politique, mais aussi la variété des formes qu'ils revêtent sur Internet, car la modification du médium entraîne la redéfinition de l'ensemble d'un genre de discours (Maingueneau, 2002 : 57).

Dans le présent article, nous nous intéresserons plus particulièrement à l'évolution de la mise en scène des émotions ${ }^{1}$ dans ce discours rituel due à la diversification de ses supports médiatiques. Les vœux, en tant que moyen verbal de manifester de la bienveillance envers les autres, semblent en effet intimement liés aux sentiments. Cependant, dans le discours politique dont la finalité est la persuasion, ceux-ci ne sont jamais gratuits. Le but ultime des vœux présentés par un politicien est de renforcer l'adhésion des citoyens à son projet politique, et l'appel aux affects n'est qu'un des moyens utilisés à cette fin. Nous nous proposons d'étudier cette fonction persuasive des émotions à partir d'exemples de messages de vœux pour l'année 2014 en adoptant l'approche de l'analyse de l'argumentation dans le discours qui appréhende l'émotion comme un moyen rhétorique (pathos) employé pour influencer l'auditoire (Amossy, $2000: 163-182$ ).

\section{VEUX PRÉSIDENTIELS EN TANT QUE MODÈLE DE VEUX DE NOUVEL AN EN POLITIQUE}

La construction des émotions dans un discours dépend du dispositif communicatif dans lequel il s'inscrit (Charaudeau, 2000 : 140-141, 2008 : 53-54). Nous caractériserons donc au préalable les vœux des hommes politiques pour la nouvelle année en tant que genre de discours en nous référant aux vœux présidentiels qui en constituent le modèle ${ }^{2}$.

Les vœux aux Français représentent un genre de discours politique par excellence selon la définition de celui-ci proposée par Teun Van Dijk : «textes écrits ou oraux produits par les hommes politiques dans un cadre institutionnel » (Van Dijk, 2003 : 13-14) [nous traduisons]. Le caractère officiel des vœux

\footnotetext{
${ }^{1}$ Nous employons le terme émotions comme terme générique désignant les états affectifs. Les mots sentiments et affects, qui n'ont pas exactement la même signification, ne seront utilisés que pour des raisons stylistiques, afin d'éviter les répétitions. Pour une analyse détaillée des relations sémantiques entre ces trois termes, voir Plantin (2011 : 5-14).

${ }^{2}$ Pour une présentation plus détaillée des vœux présidentiels en tant que genre de discours, voir p.ex. : Leblanc (2005), Ciolac (2010), Teletin (2013).
} 
présidentiels est souligné par leur mise en scène saturée de symboles nationaux. L'image du Palais de l'Élysée et la Marseillaise diffusées au début et à la fin du discours, la présence des drapeaux français et européen à côté du président de la République, le terme d'adresse « Mes chers compatriotes » et la formule finale «Vive la République, vive la France!» sont autant de marques d'un rituel politique où se manifestent l'État et l'autorité présidentielle (Leblanc, $2005: 41$ ). Telle est aussi la fonction de la forme solennelle d'un "discours-sermon » (Sauer, $2007: 229-230,266-267)^{3}$, prononcé d'un ton grave par un président qui reste debout derrière un pupitre ou est assis derrière un bureau.

Le discours de Nouvel An obéit donc à une double logique de message de vœux et de message politique si bien que la désignation vœux présidentiels peut être comprise de deux manières. D'une part, il s'agit d'une formule de politesse adressée à autrui à l'occasion du Nouvel An (voux 1 « souhaits que l'on adresse à autrui, dans diverses circonstances », TLFi) qui relève plutôt de la parole magique que politique, car l'accomplissement d'un vœu, par exemple celui de bonheur, ne dépend aucunement du locuteur (Zgółka, 2005). C'est notamment la fonction de la formule conventionnelle des vœux de bonne année qui ouvre ou clôt le discours du chef de l'État («Fidèle à une belle tradition, je présente à chacune et à chacun d'entre vous mes vœux les plus chaleureux pour la nouvelle année ${ }^{4}$ ) et de la pensée adressée aux personnes en difficulté, malades, démunis, otages, etc. ( «En cet instant, je n'oublie pas ceux qui sont dans la peine, qui vivent dans l'isolement, qui sont mal logés, qui sont même sansabri $\left.\gg^{5}\right)$.

D'autre part, le terme vœux présidentiels réfère à l'expression de la volonté politique (vøux 2 : «souhait, désir ardent de voir s'accomplir quelque chose », TLFi). En effet, quand François Hollande déclare dans son allocution de décembre 2013 : «Voilà mes vœux pour l'année nouvelle : cette bataille pour l'emploi qui doit être la réussite de la France », c'est en réalité son programme politique qu'il présente. Sur un message de vœux se greffe ainsi un message politique qui comporte le bilan de l'année écoulée et des projets d'avenir. Christoph Sauer assimile même le rôle d'un chef d'État à celui d'un barde moderne qui articule, dans son discours, les principales préoccupations de son pays (Sauer, 2007 : 230). Et s'il le fait, c'est pour assurer son peuple que la politique qu'il mène est en mesure d'y apporter une solution et de garantir au

\footnotetext{
${ }^{3}$ Christoph Sauer, auteur d'une analyse comparative des vœux de Noël et de Nouvel An des chefs d'État européens, distingue les discours-sermons (sermon-like Christmas Messages), c'est-àdire les allocutions traditionnelles, prononcées dans un cadre institutionnel, et les shows multimédias (multimedia shows) qui ont recours à une mise en scène originale (Sauer, 2007 : 229-230, 234).

${ }^{4}$ Vœux de François Hollande pour l'année 2013.

${ }^{5}$ Vœux de François Hollande pour l'année 2014.
} 
pays un bel avenir. En termes rhétoriques, les vœux présidentiels relèvent donc d'un discours épidictique qui fait l'éloge de l'action présidentielle et de la France «belle et forte dans un monde en crise» (Patier, $2011: 14$ ). Comme l'observent Chaïm Perelman et Lucie Olbrechts-Tyteca (2000: 62-72), le discours épidictique sert, au bout du compte, à " augmenter l'adhésion de l'auditoire aux valeurs qu'il exalte ». En l'occurrence, il permet de renforcer la cohésion de la nation et de réaffirmer son identité : «[...] je tiens à vous parler de ce que nous avons en commun et de plus cher : c'est-à-dire de notre pays » (vœux de François Hollande pour l'année 2014).

Le rôle des émotions dans l'allocution présidentielle correspond à son double enjeu des souhaits personnels et des souhaits politiques. Le premier lieu d'inscription de l'affectivité dans le discours est donc la séquence des vœux de bonne année et des mots de réconfort adressés aux personnes dans la peine. Désignées directement (p. ex. : joie, bonheur, souffrir, aimer) ou déclenchées par des termes à forte charge affective (p. ex. : cour, famille, solitude, maladie), les émotions évoquées correspondent essentiellement à deux champs lexicaux : celui de l'affectif et celui de la souffrance (Leblanc, 2008: 704) ${ }^{6}$. Selon le modèle de la politesse de Catherine Kerbrat-Orecchioni, cette séquence relève de la politesse positive qui consiste à valoriser l'allocutaire par des actes flatteurs pour sa face et dont le rôle est de signifier à autrui que l'on lui veut du bien (Kerbrat-Orecchioni, 2005 : 196-198). Les vœux personnels que le président adresse aux Français lui permettent donc d'exprimer sa solidarité avec les citoyens et, du point de vue de l'effet émotionnel produit sur les allocutaires, de gagner leur sympathie selon la règle de réciprocité.

La construction des émotions dans la séquence politique des vœux présidentiels est beaucoup plus complexe car soumise aux enjeux de la persuasion. Selon Patrick Charaudeau, la visée persuasive constitue le trait essentiel du discours politique qui est un discours d'influence, cherchant à «faire partager à l'autre un certain „faire croire” " et non pas à établir la vérité (Charaudeau, 2005b : 34). Dès lors, le locuteur utilise des moyens qui relèvent moins de la raison $(\log o s)$ que de l'affectivité, c'est-à-dire ceux qui tiennent à l'ethos («l'image de soi que le locuteur construit dans son discours pour contribuer à l'efficacité de son dire », Amossy, 2000 : 60) et au pathos («l'effet émotionnel produit sur l'allocutaire », Amossy, $2000: 170$ ). Ces deux moyens rhétoriques sont d'ailleurs complémentaires, car la tonalité du discours est l'une des dimensions de l'ethos (Charaudeau, 2005b : 36-37 ; Plantin, 2011 : 27).

\footnotetext{
${ }^{6}$ Par exemple : « Je pense aussi à vous qui êtes seuls et pour qui cette soirée sans personne à qui parler sera une soirée de solitude semblable à toutes les autres. Je pense à vous que la vie a éprouvés et que la tristesse ou la douleur tiennent à l'écart de la fête. »; " Je souhaite du fond du cœur que [l'année à venir] soit pour la France, pour chacun d'entre vous, pour tous ceux qui vous sont chers une année de bonheur et de réussite » (vœux de Nicolas Sarkozy pour l'année 2008).
} 
Selon Charaudeau, la mise en scène du pathos dans le discours politique repose sur un schéma dramatique propre aux contes populaires et aux récits d'aventures. Le constat initial d'un mal (diagnostic du désordre social) est suivi de l'identification de sa source (adversaire politique) et de l'intervention salvatrice d'un héros (homme politique qui tient le discours) (Charaudeau, 2005a : 69-70, 2005b : 36-37). Chacune des étapes de ce récit mobilise une autre topique du pathos parmi les trois distinguées par l'auteur: douleur/joie, angoisselespoir, antipathie/sympathie (Charaudeau, 2000 : 149-152, $2008: 51$, $55)^{7}$. Ainsi, la dénonciation d'un mal social s'appuie sur la topique de l'angoisse, la désignation du coupable repose sur la topique de l'antipathie, avec ses figures telles que l'indignation, la colère ou la haine, alors que la construction de l'image du sauveur est sous-tendue par les topiques de l'espoir et de la joie auxquelles correspondent, entre autres, les figures de la confiance, de la satisfaction et de la fierté.

Le scénario des vœux présidentiels est dominé par la séquence finale de ce schéma et orienté vers les émotions positives telles que la confiance et la fierté nationale : «La France a tous les atouts pour réussir. Nous sommes un pays d'invention, de création, dans tous les domaines. [...] Plus que jamais, il faut aimer la France. [...] Être lucide n'a jamais empêché d'être fier» (vœux de François Hollande pour l'année 2014). Si les difficultés sont mentionnées, elles sont attribuées à une cause abstraite comme la crise économique, car le président de la République, dont le rôle est de rassembler la nation, ne peut avoir recours au discours accusateur. La topique de l'angoisse sert surtout à l'expression de sa solidarité avec les citoyens et elle est tout de suite réorientée vers son opposé, à savoir la figure de la confiance : «En mai dernier, vous m'avez confié la tâche de conduire notre pays dans un moment particulièrement grave. Avec une crise historique, un chômage qui progresse implacablement depuis près de deux ans et une dette record. Je n'ignore rien de vos inquiétudes. [...] Mais ce soir, je veux vous dire ma confiance dans notre avenir [...]» (vœux de François Hollande pour l'année 2013).

\section{MISE EN SCÈNE DES ÉMOTIONS DANS LES VEUUX D’AUTRES HOMMES ET FEMMES POLITIQUES}

Comment, par rapport au modèle des vœux présidentiels, se présentent ceux d'autres acteurs de la scène politique française ? Contrairement au discours du

\footnotetext{
${ }^{7}$ Dans son article consacré aux effets de pathémisation à la télévision, Charaudeau distingue en outre la topique de l'attirance/répulsion (Charaudeau, $2000: 152-153$ ) mais il ne la reprend pas dans son analyse du pathos dans le discours politique (Charaudeau, $2008: 51,55$ ).
} 
chef de l'État, fortement ritualisé, les interventions sur Internet n'ont pas de forme fixe et varient d'un tweet de quelques mots à un film de quelques minutes. Elles forment un continuum qui s'étend entre deux modèles de vœux de Nouvel An enracinés dans la culture française : les vœux personnels de bonne année et l'allocution solennelle du président de la République. Le choix de la forme du message et les effets pathémiques visés dépendent principalement de la position idéologique et hiérarchique du locuteur dans le champ politique ${ }^{8}$.

La position idéologique de l'auteur des vœux, notamment son appartenance au parti au pouvoir ou à l'un des partis adverses, décide de la tonalité de son message. Les vœux des partisans du président épousent le registre euphorique de son discours et sont entièrement orientés vers la confiance et la fierté. En revanche, les messages des représentants de l'opposition reproduisent tout le schéma pathémique de Charaudeau (2005a: 69-70), passant de l'orientation dysphorique (critique du chef de l'État fondée sur les topiques de l'angoisse et de l'antipathie) à l'orientation euphorique (présentation de soi comme « sauveur» sous-tendue par la topique de l'espoir).

La position hiérarchique du locuteur, quant à elle, détermine surtout la forme de son message : aux dirigeants les vœux-allocutions, aux membres de partis des interventions plus modestes. Ainsi, les ministres se limitent d'habitude à poster un tweet alors que les leaders de grands partis (UMP, PS, FN) prennent pour modèle le discours présidentiel dont l'aspect solennel leur permet d'affirmer leur autorité de dirigeants et de renforcer la cohésion de leur famille politique. Leur exemple montre par ailleurs que l'ethos revendiqué par le locuteur dans son discours influe aussi sur la forme et l'orientation émotionnelle de ses vœux.

À la diversité des formes des messages de Nouvel An correspond la diversité des mises en scène des émotions. Il est toutefois possible d'en distinguer quatre catégories principales :

- la mise en scène visuelle explicite : recours à des images représentant des émotions ;

- la mise en scène visuelle implicite : recours à des images susceptibles de déclencher des émotions ;

- la mise en scène verbale explicite : emploi de «termes d'émotion » isolés (inquiétude) ou insérés dans des «énoncés d'émotion » qui lient un terme d'émotion, un lieu psychologique (un expérienceur) et une source d'émotion (je suis inquiet) (Plantin, $2011: 130,145)$;

- la mise en scène verbale implicite: emploi de termes susceptibles de déclencher des émotions (Charaudeau, $2000: 139)$ ou « formatage émo-

${ }^{8}$ Au sens que donne à ce terme Pierre Bourdieu : « champ de forces et [...] champ des luttes visant à transformer le rapport de forces qui confère à ce champ sa structure à un moment donné » (Bourdieu, $1981: 3$ ). 
tionnel » de la situation qui consiste à construire dans le discours une situation "émotionnante », culturellement associée à un type d'émotion spécifique (Plantin, $2011:$ 159, 176-181).

Précisons que dans le cas de la construction verbale des émotions, le pathos tient non seulement au contenu sémantique du message mais aussi à sa forme : moyens stylistiques, prosodiques et mimo-gestuels utilisés.

Dans notre analyse des exemples de vœux d'hommes et de femmes politiques pour l'année 2014, nous nous concentrerons sur l'expression verbale des émotions en tenant compte, tout de même, du message visuel s'il complète le sens du message verbal. En revanche, nous ne prendrons pas en considération le langage para- et non verbal qui demanderait une analyse à part. Les exemples retenus représentent un continuum allant de la forme minimale des simples vœux de bonne année à la forme maximale des vœux-allocutions.

\subsection{VEUX DE NOUVEL AN COMME MESSAGE PERSONNEL : NICOLAS SARKOZY, JEAN-LUC MELENCHON, CHRISTIANE TAUBIRA, BERNARD DELANOË}

La forme minimale des vœux personnels et apolitiques semble la moins fréquente. Citons toutefois l'exemple du message de Nicolas Sarkozy, publié sur Facebook, qui met en scène des émotions relevant de l'intime, comme l'amitié et la tendresse, bien que le locuteur s'adresse à ses partisans pour les remercier de leur soutien :

(1) Mes chers Amis,

Très belles fêtes à chacun d'entre vous !

Durant cette année 2013, j'ai pu compter une nouvelle fois sur votre soutien, votre amitié et votre fidélité. Je vous en suis extrêmement reconnaissant et soyez certains que je serai toujours là parmi vous. Je vous souhaite de passer ces moments de fin d'année avec vos proches, d'aller à l'essentiel en profitant de chaque instant de vie offert par la proximité et la tendresse de ceux que vous aimez.

Avec toute mon affection. NS.

L'orientation euphorique de ce message est construite à l'aide de termes et d'énoncés d'émotion (amitié, affection, tendresse, être extrêmement reconnaissant) et de mots à forte charge affective (amis, fidélité, vos proches, proximité). L'absence apparente de toute émotion politique est déterminée par l'ethos que l'ancien président revendique depuis la fin de son mandat: celui de politicien retiré de la vie publique. Le choix du registre intime et du moment de la publication de ses vœux - Noël au lieu de la date consacrée du 31 décembre renforcent son ethos de personne privée. Il n'empêche que le pathos construit dans les vœux de Sarkozy sert à s'assurer la fidélité de ses partisans politiques. 
À l'opposé de ce message empreint d'ambiance de Noël qui occulte sa visée politique, se situent les « anti-vœux » de Jean-Luc Mélenchon dont la dimension festive disparait au profit de la colère politique :

(2) Mes vœux pour cette année : qu'ils s'en aillent tous. Viennent les jours heureux et le temps des cerises!

L'orientation émotionnelle des vœux est ainsi inversée: un acte essentiellement flatteur pour la face de l'allocutaire se transforme en un acte menaçant. Là aussi, le choix de l'émotion mise en scène est déterminé par l'ethos endossé par le locuteur, celui d'un homme politique qui refuse tout compromis et est connu pour son franc-parler.

Ces deux cas restent cependant isolés, car, d'habitude, les deux aspects du message de vœux de Nouvel An - festif et politique - sont associés. Ainsi, dans le tweet de Christiane Taubira, la formule conventionnelle des vœux de bonne année est précédée d'un bref bilan émotionnel de l'année passée qui fait allusion aux attaques racistes dont la ministre de la justice a été victime et aux réactions violentes suscitées par la loi sur le droit au mariage pour tous dont elle a porté le projet à l'Assemblée Nationale :

(3) Quelle année! Joies inattendues, émotions soudaines comme pluies d'Amazonie, colères, et vous, là, toujours. Belle année nouvelle. ChT.

Les vœux deviennent un prétexte pour revivre les émotions vécues et les partager avec ses partisans (et vous, là, toujours). La mise en scène émotionnante fait surtout ressortir l'intensité des affects par l'association des termes d'émotion opposés joies et colères, l'emploi d'adjectifs qui suggèrent le caractère inattendu du stimulus (inattendues, soudaines) et le recours à de multiples procédés d'intensification: phrase exclamative, phrase sans verbe comportant une énumération, comparaison à valeur intensive (soudaines comme pluies d'Amazonie). Comme dans le message de Nicolas Sarkozy, l'évocation des émotions communes vise à renforcer le lien affectif avec ses partisans.

Si l'identité politique de Christiane Taubira influence la construction du pathos dans la partie bilan de ses vœux, celle de Bertrand Delanoë détermine l'effet pathémique de la partie perspectives d'avenir où le maire de Paris s'adresse à sa ville personnifiée :

(4) À chacune et chacun d'entre vous, je souhaite le meilleur en 2014. Et à Paris, de rester une ville toujours confiante en son avenir. \# Bonne Année.

Bien que ses vœux n'évoquent explicitement qu'une seule émotion, la confiance, leur charge émotionnelle, due au contexte des élections municipales 2014, est plus importante qu'il n'y paraît. Il s'agit, en effet, des derniers vœux 
que Delanoë présente aux Parisiens en tant que maire de la capitale et qui rappellent donc une formule d'adieu. Cette impression vient de l'allusion à l'avenir de Paris et du choix de l'adverbe toujours qui dépasse la perspective d'une année, horizon temporel habituel des vœux de Nouvel An. Le côté nostalgique de ce message est de plus renforcé par sa forme manuscrite, à l'ancienne. Par ailleurs, cette touche personnelle ainsi que l'individualisation des vœux adressés «à chacune et chacun» sont des marques de politesse positive qui traduisent le respect et peut-être aussi une certaine affection que Delanoë a pour les habitants de Paris.

\subsection{VEUUX DE NOUVEL AN COMME PROJET POLITIQUE : NATHALIE KOSCIUSKO-MORIZET}

Si dans le message du maire de Paris, l'influence du contexte politique sur les effets pathémiques de ses vœux reste implicite, dans l'exemple suivant elle devient directe. Il s'agit toujours de la perspective des élections municipales qui transforme les vœux de Nathalie Kosciusko-Morizet, candidate UMP à la mairie de Paris, en une vidéo de campagne. La politicienne présente ses vœux assise à table dans un bistrot à Paris (séquences initiale et finale du film) ce qui détermine d'emblée son identité de Parisienne et de candidate proche de ses électeurs. Cet ethos de solidarité (Charaudeau, 2005a: 125-128) de femme politique «de terrain», attentive aux besoins des habitants de la capitale est construit déjà dans les deux premières phrases de son discours grâce au recours au champ lexical du contact humain et à l'emploi du verbe de sentiment ressentir :

(5) Tout au long de cette année 2013, je suis allée à la rencontre des Parisiennes et des Parisiens pour écouter, pour partager, pour proposer. Dans ces nombreux échanges avec eux, avec vous, j'ai ressenti un ardent besoin, celui d'une nouvelle espérance.

Mais c'est surtout le message visuel qui véhicule cette image de proximité avec les électeurs. Les photos diffusées tout au long du film mettent en scène l'émotion partagée de la candidate UMP et des Parisiens. Elles représentent la politicienne, toujours souriante, en train de parler avec des Parisiens de différents groupes sociaux (petits commerçants, musulmans, handicapés, sansabri, etc.) ou prenant des bains de foule.

La construction des émotions dans le message verbal est plus complexe et s'appuie sur le scénario pathémique de Charaudeau (2005a : 69-70) : diagnostic du désordre social, désignation du coupable, intervention salvatrice de NKM. La séquence qui véhicule des émotions négatives est cependant glissée entre les 
passages orientés vers des émotions positives pour que la tonalité globale du message reste euphorique ${ }^{9}$. Ainsi, la première émotion mise en scène tout au début du discours est l'espoir désigné par le terme espérance. Employé dans l'expression ressentir le besoin d'une nouvelle espérance, ce vocable semble autant un terme d'émotion que le synonyme du mot changement qui, monopolisé par François Hollande, ne peut plus être utilisé par la candidate de droite.

Cette première séquence euphorique est suivie d'un passage dysphorique qui justifie le besoin de changement en dressant le constat du déclin du pays, résultat de la politique menée par la gauche. La stratégie pathémique employée est celle $\mathrm{du}$ « formatage émotionnel » de la situation (Plantin, 2011: 159) :

(6) Cette nouvelle espérance, c'est la meilleure réponse à la politique menée par François Hollande qui aggrave chaque jour les inégalités et la première d'entre elles, le chômage. Il ne veut rien entendre, il ne veut rien changer, il nous entraine dans le déclassement et le déclin. Paris ne doit pas rester le laboratoire de toutes les erreurs socialistes. Des impôts qui explosent, une dette multipliée par quatre, la sécurité qui chaque jour se dégrade, les classes moyennes qui doivent partir. Et surtout une ville qui attire moins, qui n'innove plus, qui perd de sa lumière. C'est la fin d'un cycle.

Le discours critique actualise d'abord la topique de l'angoisse. Les émotions ne sont pas nommées, mais connotées par le champ lexical du déclin (substantifs : déclin, déclassement, fin d'un cycle ; verbes désignant des procès négatifs : aggraver, entraîner dans, se dégrader, attirer moins, perdre) et celui des injustices sociales (inégalités, hausse des impôts, chômage). La seconde topique mobilisée est celle de l'antipathie. Les responsables des maux français sont directement désignés: François Hollande et la gauche, parti d'Anne Hidalgo, concurrente de NKM aux élections municipales. Le discours accusateur attribue au président une attitude de mépris (il ne veut rien écouter) ainsi que des actes d'injustice (qui aggrave chaque jour les inégalités) et destructeurs (il nous entraîne dans le déclassement et le déclin). La dénonciation de la politique des socialistes a pour but de susciter la colère et l'indignation. Selon Aristote, la première est en effet dirigée contre ceux qui nous méprisent et outragent alors que la seconde est liée à un sentiment d'injustice (Rhétorique 1378b-1380a, 1386b-1387b). Les deux sont considérées comme des émotions politiques par excellence qui incitent à l'action (Danblon, 2005 : 177). Tel est aussi leur rôle dans le discours de la candidate UMP qui, dans la séquence suivante, invite les Parisiens à adhérer à son projet politique. Ses vœux retrouvent leur orientation euphorique vers l'espoir :

${ }^{9}$ La tonalité dysphorique de cette séquence est de plus atténuée par le message visuel qui continue à développer la topique de la joie, actualisée par des scènes de convivialité entre NKM et les Parisiens. 
(7) Oui, c'est ici, à Paris, que tout peut changer. C'est ici que nous préparons une majorité d'alternance pour une nouvelle gouvernance [...]. Pour les Parisiennes et les Parisiens. Pour que Paris soit toujours Paris. Pour que chacun y trouve l'énergie nécessaire pour réaliser ses rêves. Paris doit donner plus d'énergie à ses habitants qu'elle ne leur en prend, c'est ma conviction mais c'est aussi mon engagement, celui que je prends devant tous les Parisiens, si je suis élue maire de Paris en mars prochain.

La construction des émotions dans le message de Nathalie KosciuskoMorizet est donc entièrement soumise à sa finalité politique. La candidate ne présente ses vœux de bonne année que vers la fin du film et ce pour enchaîner tout de suite sur l'actualité électorale et appeler de nouveau à voter pour elle :

(8) Je souhaite à chacun d'entre vous mes vœux les plus sincères de bonheur à partager avec ceux que vous aimez [...]. En 2014, tout se joue à Paris. En 2014, ce sont les Parisiens qui écrivent leur histoire. Ensemble, faisons de 2014 l'année des Parisiens.

\subsection{VEUUX-ALLOCUTIONS DES CHEFS DE GRANDS PARTIS : HARLEM DÉSIR ET JEAN-FRANÇOIS COPÉ}

Les deux derniers exemples de vœux seront analysés ensemble, car ils sont construits en miroir et reposent sur la même stratégie de la mise en scène du pathos à travers le «formatage émotionnel» de la situation présentée dans le discours (Plantin 2011 : 159). Il s'agit des allocutions enregistrées des leaders des deux plus grandes forces politiques de France : Harlem Désir (PS) et JeanFrançois Copé (UMP) $)^{10}$. Les deux adoptent le modèle des vœux présidentiels, celui d'un discours-sermon, prononcé debout, dont la forme solennelle leur permet d'affirmer leurs ethos de chefs (Charaudeau, 2005a : 118-125). Parmi d'autres éléments empruntés au rituel présidentiel, citons encore le terme d'adresse "Mes chers compatriotes de métropole, d'outre mer, de l'étranger » employé par Désir et les drapeaux français et européen qui font partie du décor de l'intervention de Copé. Les deux discours comportent également les vœux de bonne année :

(9) H. D. : Avec l'ensemble des Socialistes, [...] je veux non seulement vous adresser mes vœux les plus chaleureux de bonheur et de santé mais également souhaiter le meilleur pour chacun et pour notre pays pour la nouvelle année.

(10) J.-F. C. : Mes chers amis, à chacun d'entre vous, à vos familles, à vos proches, je souhaite une très belle année 2014. Je vous souhaite de la joie, de la paix et de l'amour.

${ }^{10}$ Les deux hommes politiques ont depuis quitté la tête de leur parti : Harlem Désir en avril 2014 et Jean-François Copé un mois plus tard. 
une pensée pour les personnes en détresse :

(11) H. D. : Mes premières pensées vont ce soir à ceux qui sont dans la solitude, dans la précarité ou la maladie, à tous ceux qui affrontent les difficultés.

(12) J.-F. C. : Je veux avoir une pensée particulière pour ceux qui sont frappés par le chômage ou les difficultés sociales, pour ceux qui souffrent de la solitude ou de la maladie.

et un appel à combattre la haine sociale :

(13) H. D. : Dans ces deux élections, nous devrons combattre l'extrémisme qui attise les haines et les conservateurs qui veulent s'opposer au progrès et à la solidarité.

(14) J.-F. C. : Enfin, je voudrais mettre en garde contre la banalisation de la haine. Contre le racisme. Contre toutes les formes de racisme, d'antisémitisme, d'islamophobie, de xénophobie...

Enfin, à l'instar du président de la République, les deux leaders adressent aux allocutaires un message de confiance dans l'avenir du pays. Mais cette notion ne reçoit pas la même interprétation dans les discours des chefs des partis aux idéologies opposées.

Harlem Désir s'adresse à tous les Français au nom du parti au pouvoir. Adhérant à la politique du chef de l'État, il emploie le pronom de la $1^{\text {re }}$ personne du pluriel nous et épouse le ton optimiste des vœux présidentiels : «Et c'est au nom de cette idée d'une France plus forte parce que plus solidaire que depuis dix-huit mois, aux côtés de François Hollande, nous menons une politique de progrès $[\ldots]$ ». Son message s'appuie sur la topique de la joie, et plus précisément sur les figures de la satisfaction et de la fierté. Seul ce dernier sentiment est toutefois affiché dans un énoncé d'émotion explicite: «Nous sommes fiers d'une France à nouveau écoutée et respectée dans le monde [...]. Nous sommes fiers d'une France universaliste, qui agit pour la sécurité et la solidarité internationales $[\ldots] »$. Le sentiment de satisfaction est, quant à lui, induit du bilan positif de l'action du gouvernement. Désir énumère les mesures adoptées en 2013 (100000 emplois d'avenir, extension de la couverture maladie universelle, priorité à l'éducation) en se référant aux valeurs à forte charge affective telles que la justice, la solidarité, l'égalité, le progrès : «Nous assumons notre combat contre les inégalités, notre choix du tout-pour-l'emploi, nous assumons d'avoir replacé la justice sociale et la solidarité au cœur de notre société ». Les problèmes sociaux qui restent à résoudre sont, en revanche, désignés par des termes euphémiques. Par exemple, le terme négatif chômage est remplacé par le terme neutre emploi : «Ce grand débat démocratique doit porter sur les enjeux concrets de notre vie collective. [...] En Europe, c'est la croissance, l'emploi $[\ldots] »$. 
L'ensemble du message du premier secrétaire du PS est donc orienté vers des émotions positives et a pour objectif de renforcer l'adhésion des allocutaires à la politique de la gauche au pouvoir et plus particulièrement à les inciter à voter pour les socialistes aux élections municipales et européennes en 2014 : « Nous voulons rassembler une nouvelle majorité progressiste au Parlement européen $[\ldots] »$.

Les échéances électorales se trouvent aussi au cœur du message du leader de l'opposition, mais son enjeu est la quête du pouvoir. Si Harlem Désir s'adresse à tous les Français en tant que dirigeant du parti dominant, Jean-François Copé ne parle qu'à sa famille politique (« Mes chers amis ») dont il souhaite resserrer les rangs. Pour inciter les militants UMP à se rendre aux urnes, il utilise la même stratégie du discrédit du président de la République que Nathalie KosciuskoMorizet. Il dresse un tableau noir de la France pour désigner François Hollande comme coupable (« Face à cette situation dramatique, je demande au président de la République de prendre acte de son échec devant les Français [...]») et pour appeler finalement au « vote sanction ».

Au discours de l'éloge de Désir correspond ainsi le discours du blâme de Copé. Si le premier se dit fier de la France, le second se dit inquiet: "Je suis inquiet de voir la communauté nationale se fissurer, avec des territoires entiers abandonnés par l'État». L'inquiétude, voire la peur sont aussi attribuées aux Français : "Les familles, les classes moyennes sont touchées par la peur du déclassement $[\ldots] »$. À la topique de la joie se substitue la topique de l'angoisse évoquée par :

- des termes désignant des maux sociaux : un troisième fléau qui menace ; le matraquage fiscal sans précédent ; l'appauvrissement, la précarisation des millions de Français ; la baisse du pouvoir d'achat ; etc. ;

- le champ lexical du déclin : la France [...] est affaiblie ; notre pays étouffé sous le poids du fardeau socialiste; les entreprises sont écrasées par la multiplication des taxes, des normes, des contrôles et des suspicions; les réglementations absurdes qui nous paralysent; nos compatriotes qui sont déboussolés ; etc. ;

- des adjectifs intensifieurs: cette situation dramatique; cette crise profonde; une très forte progression du chômage ; les rafistolages calamiteux et ruineux; etc.

La dramatisation des faits et les émotions négatives qu'elle suscite servent, au bout du compte, à attiser l'ardeur politique des militants de droite : «Dans cette crise profonde, je veux vous le dire, notre famille politique n'a pas le droit de se dérober devant ses responsabilités ». Car le «salut de la France» ne peut venir que de la part de l'UMP. Tel est le sens du message de confiance dans les vœux de Jean-François Copé. Le discours du leader de la droite reflète, comme un miroir déformant, le discours du leader de la gauche. 
Entre bilan de l'année écoulée et perspectives d'avenir, l'objectif des messages de Nouvel An publiés sur Internet par des hommes et des femmes politiques est le même que celui des vœux présidentiels : renforcer la cohésion de la communauté à laquelle l'on s'adresse et lui redonner confiance. Les messages diffusés en ligne diffèrent cependant par leur teneur, allant jusqu'à contredire l'esprit des vœux et désacraliser le rituel de l'allocution télévisée du président de la République. De la colère à l'affection, de la peur du déclin de la France à la fierté nationale, toute une gamme affective est développée pour s'assurer la fidélité de ses partisans. Dans le discours politique, soumis aux enjeux de la persuasion, aucune émotion ne peut rester gratuite. 


\section{BIBLIOGRAPHIE}

Amossy, R. (2000): L'argumentation dans le discours. Paris : Nathan.

ARYSTOTELES (1988): Retoryka - Poetyka. Warszawa: PWN.

BouRdieu, P. (1981): " La représentation politique [Éléments pour une théorie du champ politique]». Actes de la recherche en sciences sociales, 36-37, février/mars 1981, La représentation politique-1, 3-24.

Charaudeau, P. (2000): «Une problématisation discursive de l'émotion. À propos des effets de pathémisation à la télévision ». In : Ch. Plantin, M. Doury, V. Traverso (éds), Les émotions dans les interactions. Lyon : Presses universitaires de Lyon, 125-155.

Charaudeau, P. (2005a): Le discours politique. Les masques du pouvoir. Paris : Vuibert.

Charaudeau, P. (2005b): "Quand l'argumentation n'est que visée persuasive. L'exemple du discours politique ». In : M. Burger, G. Martel (éds), Argumentation et communication dans les médias. Québec : Éd. Nota bene, 29-49.

Charaudeau, P. (2008): « Pathos et discours politique ». In : M. Rinn (éd.), Émotions et discours. L'usage des passions dans la langue. Rennes: Presses universitaires de Rennes, 49-58 [en ligne]. URL: <http://www.patrick-charaudeau.com/Pathos-et-x.html>. (Consulté le 15 mars 2014).

Ciolac, M. (2010): « Vœux présidentiels télédiffusés dans deux États de l'Union européenne ». Signes, Discours et Sociétés, 5 [en ligne]. URL : <http://www.revue-signes.info/document. php?id=1622>. (Consulté le 10 mars 2014).

Danblon, E. (2005): La fonction persuasive. Anthropologie du discours rhétorique : origines et actualité. Paris : Armand Colin.

Kerbrat-OrecChIONI, C. (2005): Le discours en interaction. Paris : Armand Colin.

LeBlanc, J.-M. (2005): Les voux présidentiels sous la Cinquième République (1959-2001). Recherches et expérimentations lexicométriques à propos de l'ethos dans un genre discursif rituel. Thèse de Doctorat en Sciences du Langage, Université de Paris 12 Val-de-Marne [en ligne]. URL : 〈http://leblanc.jeanmarc.free.fr/These/ThseJM_Leblanc.pdf〉. (Consulté le 10 mars 2014).

LEBLANC, J.-M. (2008): «Une approche expérimentale du rituel politique, l'analyse récursive des énoncés sous Alceste ». In : S. Heiden, B. Pincemin (éds), JADT'08: Actes des $9^{\text {es }}$ Journées internationales d'Analyse statistique des Données Textuelles, Lyon, 12-14 mars 2008. Vol. 2. Lyon : Presses universitaires de Lyon, 701-712 [en ligne]. URL : <http://lexicometrica.univparis3.fr/jadt/jadt2008/pdf/leblanc.pdf $>$. (Consulté le 10 mars 2014).

Maingueneau, D. (2002): Analyser les textes de communication. Paris : Nathan.

PATIER, X. (2011): «Du verbe à l'image ». In : Bonne année, la France! Les voux présidentiels depuis 1958. Paris : La Documentation française, 9-14.

Perelman, C., Olbrechts, L. (2000): Traité de l'argumentation. La Nouvelle Rhétorique. $5^{\mathrm{e}}$ éd. Bruxelles : Éd. de l'Université de Bruxelles.

Plantin, CH. (2011): Les bonnes raisons des émotions. Principes et méthodes pour l'étude du discours émotionné. Berne : Peter Lang.

SAUER, CH. (2007): "Christmas Messages by heads of state. Multimodality and media adaptations". In : Anita Fetzer, Gerda Eva Lauerbach (éds), Political discourse in the media : crosscultural perspectives. Amsterdam/Philadelphia: John Benjamins Publishing Company, 227-273.

Teletin, A. (2013): « Les vœux présidentiels au Portugal, en France et en Roumanie, et la crise internationale. Les enjeux des formes d'adresse et des procédés d'atténuation/intensification ». Mots. Les langages du politique, 101, 31-46. 
TLFi. Trésor de la langue française informatisé. URL : 〈http://atilf.atilf.fr/>.

VAN DiJK, T. A. (2003): „Dyskurs polityczny i ideologia”. Etnolingwistyka, 15, 6-28.

ZGÓŁKA, T. (2005): „Życzenie jako kategoria językowo-komunikacyjna”. In : A. Dąbrowska, A. Nowakowska (éds), Życzliwość w języku i kulturze. Język a Kultura. Vol. 17. Wrocław: Wydawnictwo Uniwersytetu Wrocławskiego, 17-22.

\section{Corpus}

\section{Vœux présidentiels}

Vœux de Nicolas Sarkozy pour l'année 2008: URL : <http://discours.vie-publique.fr/notices/ 087000012.html>. (Consulté le 2 juin 2014).

Vœux de François Hollande pour l'année 2013 : URL : <http://www.elysee.fr/declarations/article/ v-ux-aux-francais/>. (Consulté le 2 juin 2014).

Vœux de François Hollande pour l'année 2014 : URL : <http://www.elysee.fr/declarations/article/ v-ux-aux-francais-5/>. (Consulté le 2 juin 2014).

\section{Vœux des hommes et des femmes politiques pour l'année 2014}

Vœux de Nicolas Sarkozy : URL : <https://fr-fr.facebook.com/nicolassarkozy/posts/10151796493 856078 ? fref $=$ nf $>$. (Consulté le 2 juin 2014).

Vœux de Jean-Luc Mélenchon : URL : <https://twitter.com/JLMelenchon/status/41819633767574 $3233>$. (Consulté le 2 juin 2014).

Vœux de Christiane Taubira : URL : <https://twitter.com/ChTaubira/status/418097970916388864〉. (Consulté le 2 juin 2014).

Vœux de Bertrand Delanoë : URL : < https://twitter.com/BertrandDelanoe/status/4180393105424 71168/photo/1>. (Consulté le 2 juin 2014).

Vœux de Nathalie Kosciusko-Morizet: URL : <http://www.nkmparis.fr/presse/communiques/ voeux-parisiennes-parisiens>, URL : <http://www.dailymotion.com/video/x192ou0_voeuxde-nkm-aux-parisiennes-et-aux-pari siens_news $>$. (Consulté le 2 juin 2014).

Vœux d'Harlem Désir : URL : <http://www.parti-socialiste.fr/articles/harlem-desir-souhaite-tousles-francais-une-belle-et-heureuse-annee-2014>. (Consulté le 2 juin 2014).

Vœux de Jean-François Copé : URL : <http://www.u-m-p.org/actualites/a-la-une/voeux-de-jeanfrancois-cope-pour-lannee-2014-102973012>. (Consulté le 2 juin 2014). 